\title{
Abdominoscrotal Haematocele in an Adult and Its Successful Treatment
}

"Uday S. Kumbhar, Oseen Shaikh, Sandeep Bhattarai

\begin{abstract}
Abdominoscrotal hydrocele (ASH) is a variant of hydrocele that rarely occurs in adults. ASH has two sacs, one in the scrotum and one in the abdomen connected through the inguinal canal. Abdominoscrotal haematocele is a rare complication of ASH. We report a 57-year-old male patient who presented to a tertiary care hospital in Puducherry, India, in 2019 with complaints of swelling in the scrotum for 15 years and abdominal pain for two months. Both the swellings were soft and cross fluctuation was present. Imaging confirmed the diagnosis of ASH. During a diagnostic laparoscopy, the abdominal sac was decompressed and found to have thick brownish fluid suggestive of haematocele. It was demonstrated that both sacs were connected. Due to difficulty in the dissection of the sac, the procedure was converted to an open procedure. Both the sacs were excised and Lytle's repair was done for the dilated internal ring. The patient recovered and no recurrence of any swelling in the abdomen or scrotum was found at the end of a one-year follow-up.
\end{abstract}

Keywords: Testicular Hydrocele; Scrotal Hydrocele; Testicular Haematocele; Scrotal Haematocele; Laparoscopic Surgical Procedure; Laparoscopic Surgery; Case Report; India.

A BDOMINOSCROTAL HYDROCELE (ASH) IS defined as hydrocele extending into the abdominal cavity forming two intercommunicating compartments. ${ }^{1} \mathrm{ASH}$, also called hydrocele en-bisac, is a rare variant of a hydrocele that can occur commonly in the paediatric age group but is rarely seen in the adult population. Patients are usually asymptomatic or present with variable sized abdominoscrotal swelling. ASH can become complicated due to bleeding inside the sac, which can occur due to trauma, malignancy or be spontaneous leading to the formation of haematocele. ${ }^{2}$ The exact aetiology of ASH is not known, but various theories have been proposed. These theories explain disease occurrence either as an extension of the scrotal sac into the abdomen or abdominal sac into scrotum or both. It is diagnosed clinically, and imaging is used to support the diagnosis. ${ }^{3}$ Treatment can be conservative or surgical. In adults, treatment is done by surgical means, either via an open or laparoscopic approach.

\section{Case Report}

A 57-year-old male patient presented to a tertiary care hospital in Puducherry, India, in 2019 with left-sided scrotal swelling for the previous 15 years and abdominal pain for the past two months. There were no other symptoms such as altered bowel or bladder habits. The patient was a non-alcoholic, non-smoker with no comorbidities. On examination, there was a large leftside scrotal hydrocele and large left-lower abdominal swelling [Figure 1]. Abdominal swelling was soft, nontender and had a smooth surface. Scrotal swelling was fluctuant; there was a cross fluctuation between the abdominal and scrotal swelling. The transillumination test was negative in the scrotal swelling.

Ultrasonography of the abdomen and scrotum showed a large hypoechoic collection with internal echoes $>20 \mathrm{~cm}$ in size in the left-lower abdomen with extension into the left inguinoscrotal region with normal bilateral testis. Contrast-enhanced computed tomography of the abdomen showed a large hypodense retroperitoneal cystic lesion measuring 24 $\times 16 \mathrm{~cm}$ in the entire left side of the abdomen with the craniocaudal extent from the left lobe of the liver up to the pelvic brim. Anteriorly it was extending up to the anterior abdominal wall displacing entire intraperitoneal structures to the right side. Another hypodense cystic lesion was present in the left hemiscrotum measuring $18 \times 9 \mathrm{~cm}$ with few septations and communication with the abdominal cyst which was diagnostic of ASH [Figure 2]. The bilateral testis appeared normal; however, the size of the left testis was a little smaller than the right.

Subsequently, a diagnostic laparoscopy was done. Pneumoperitoneum was created by open technique with a $10 \mathrm{~mm}$ trocar in the right lumbar region. Two working ports were used; one $5 \mathrm{~mm}$ trocar was placed in the right lumbar region $7 \mathrm{~cm}$ above and lateral to the umbilicus and another $10 \mathrm{~mm}$ trocar was placed $6 \mathrm{~cm}$ below the umbilicus in the midline. There was a large retroperitoneal cystic lesion occupying most of the left half of the abdomen. It had displaced the sigmoid and descending colon medially. Craniocaudally it extended from the left lobe of the liver up to the pelvic brim. 


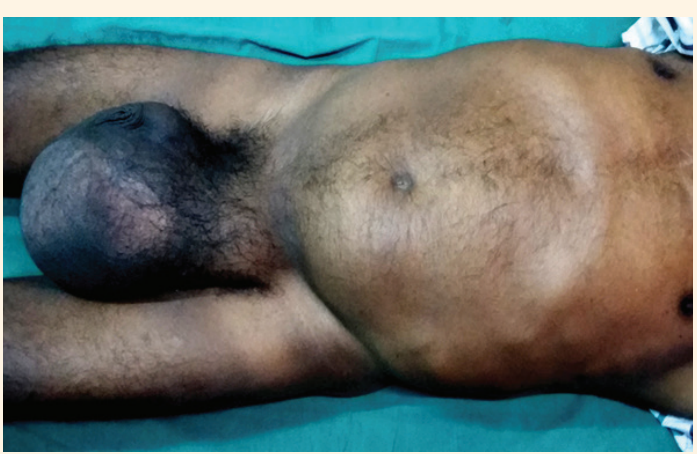

Figure 1: Photograph of a 57-year-old male patient showing abdominal and scrotal swelling.

Inferiorly the cyst extended into the inguinal canal through the deep inguinal ring. Due to the large size of the cyst and inadequate access for the dissection, a decision was made to decompress the cyst. Initially, the cyst was punctured using a Veress needle which was inserted transcutaneously. It was not possible to do an effective aspiration as the fluid was thick and brown to greenish in colour. The Veress needle was replaced with a $5 \mathrm{~mm}$ trocar and 1,500 $\mathrm{mL}$ of fluid was aspirated. Another $10 \mathrm{~mm}$ trocar was placed through the bottom of the scrotum into the hydrocele sac [Figure 3] and $650 \mathrm{~mL}$ of the fluid was drained which had the same characteristics as the abdominal fluid. There were two openings at the cranial pole of the sac, one of which was communicating with the abdominal cyst [Figure 3]. The retroperitoneal cyst was dissected from the lateral abdominal wall. Once the dissection proceeded medially, it was impossible to separate the cyst from the sigmoid colon and its mesentery. Due to the risk of inadvertent injury, the decision was made for open surgery conversion. The retroperitoneal cyst was approached transabdominally using a left inguinal incision. The cyst was opened and brownish muddy

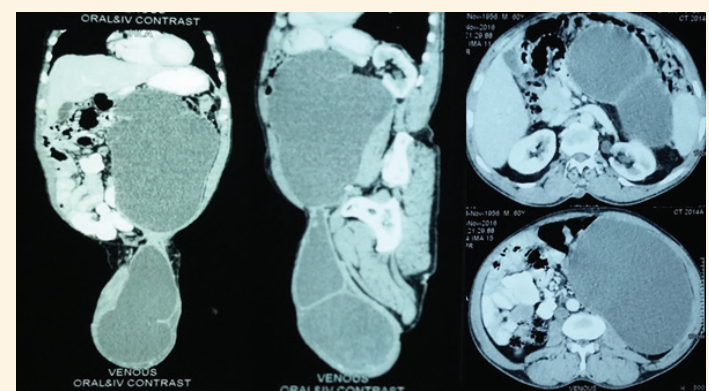

Figure 2: Contrast-enhanced computed tomography images showing large hypointense-walled collection of $24 \times 16 \mathrm{~cm}$ in the left side of the retroperitoneum extending into the ipsilateral inguinoscrotal region measuring $18 \times 9 \mathrm{~cm}$

fluid was evacuated. The cyst wall was completely excised; it was extending into the scrotum through the inguinal canal. A separate vertical incision was made over the scrotum. The hydrocele sac was opened and the contents were found to be similar to those in the abdomen, suggestive of haematocele. In addition, the testis had haematomas. A decision was taken for a left orchidectomy. Both the abdominal and scrotal sacs were dissected and the communication between the two sacs was well demonstrated [Figure 3]. Complete excision of both the sacs along with the intercommunicating tract was done. The internal ring was mildly dilated; however, there was no weakening of the abdominal wall. Hence, Lytle's repair was done for the dilated internal ring. Subsequently, the port sites, inguinal and scrotal incisions were closed.

The patient was discharged after eight days without any complication. The patient was under regular follow-up. Operative site wounds healed completely, and the scrotum regained its normal size [Figure 4]. No recurrence of any swelling in the abdomen or scrotum at the end of a one-year follow-

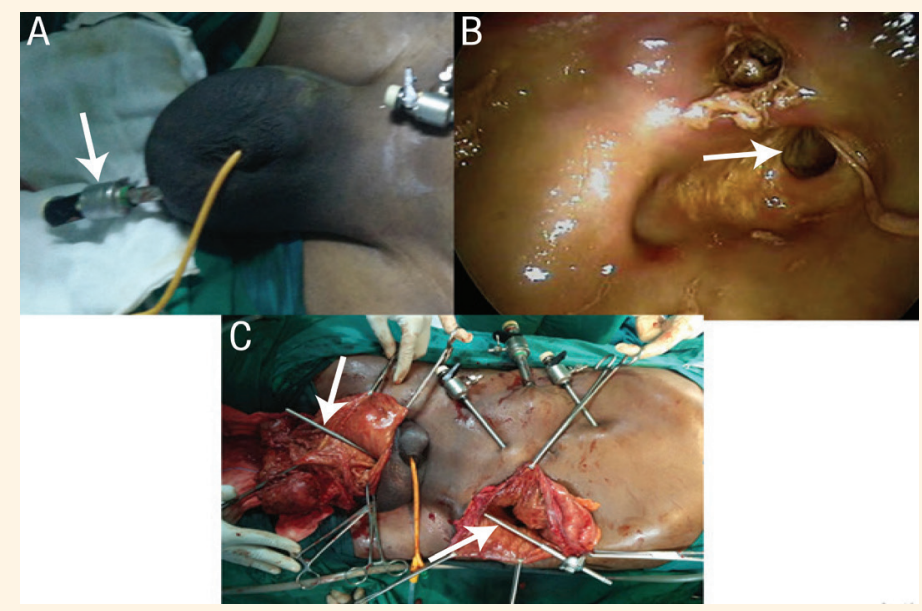

Figure 3: Intraoperative images showing (A) scrotal trocar (arrow) and (B) trans-scrotal endoscopic view of the scrotal sac with the opening of communication (arrow) with abdominal sac as well as (C) communication between abdominal sac and scrotal sac by passage of laparoscopic suction cannula (arrows). 


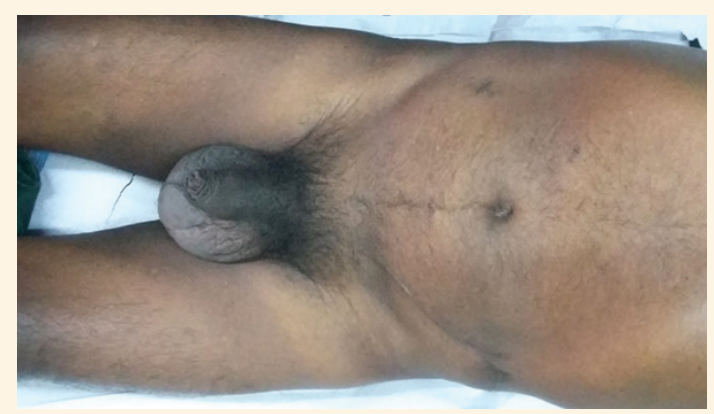

Figure 4: Photograph of the patient taken one month after the surgery during follow-up showing healed port sites, inguinal and scrotal scars.

up was found. Written informed consent was obtained from the patient to use his images for publication purposes.

\section{Discussion}

Hydrocele is an accumulation of fluid between the layers of tunica vaginalis. It can occur in infants or adults. An infantile hydrocele is due to persistent patent processus vaginalis. Adult or vaginal hydrocele occurs when fluid collection occurs between tunica vaginalis, where processus vaginal is obliterated. A variant of hydrocele, called "hydrocele of the cord" occurs due to partial closure of processus vaginalis. ASH is a rare variant of hydrocele characterised by scrotal hydrocele extending into the abdomen as cystic abdominal swelling through the inguinal canal.

$\mathrm{ASH}$ is defined as a hydrocele extending into the abdominal cavity forming two intercommunicating compartments. ${ }^{1}$ Dupuytren first described this in 1834 and it was called Dupuytren hydrocele or hydrocele en-bisac. ${ }^{4}$ In 1919, Bickle proposed a new term for hydrocele en-bisac called abdominoscrotal hydrocele. ${ }^{5}$

$\mathrm{ASH}$ is a rare entity and has been reported both in infants and old age. It is seen more commonly in the paediatric age group than adults. Overall, in adults, there are less than 250 cases reported in the literature. India has the highest incidence of ASH in the world. ${ }^{3}$ Literature review suggests that ASH is more common on the right side than the left. ASH is usually a congenital anomaly; it starts from birth as an inguinoscrotal swelling that extends to the abdomen. It may be missed in childhood and may present in adult life. Sac of ASH extends through the inguinal canal and this part of the sac acts as an isthmus between the two sacs giving a dumbbell shape appearance. ${ }^{2}$ Interestingly, the current patient presented with predominant left-sided scrotal swelling and nonspecific abdominal pain.
The abdominal sac can be retroperitoneal or preperitoneal. The sac is usually covered with the transversalis fascia. The abdominal sac may be of variable size and shape and can be as large as a football. ${ }^{1}$ Although the scrotal sac is of varying size, it is usually small. These sacs can be unilocular or multilocular. There is intercommunication between the two sacs without any communication with the peritoneal cavity.

There are various theories proposed for the development of ASH. Dupuytren hypothesised that ASH occurs due to an extension of the scrotal sac into the abdomen through the inguinal canal whenever the scrotal sac is under high pressure. ${ }^{1}$ Jacobson hypothesised that ASH occurs as a result of an extension of infantile hydrocele. ${ }^{6}$ Others added another concept to this hypothesis: the coexistence of inguinal defect and infantile hydrocele. ${ }^{7}$ Roller hypothesised that encysted hydrocele of cord and ordinary hydrocele might form ASH; however, they did not explain the intercommunication between the sacs. ${ }^{8}$ Some other theories have been proposed but these have been less accepted. ASH can occur due to an extension of the preformed peritoneal sac into the scrotum through the inguinal canal and/or due to the flow of fluid downwards through persistent processus vaginalis, which acts as a one-way valve. ${ }^{1,9}$

ASH occurring in the paediatric age group is known to have spontaneous regression, but such resolution has not been noted in adults. The patient presents with painless inguinoscrotal swelling and abdominal swelling which may be variable in size. Scrotal swelling may be reducible or partially emptied in the supine position. Cross-fluctuation may be present due to the intercommunication of the two sacs, which is diagnostic for ASH. The presence of 'springing back ball sign' and hourglass transillumination are diagnostic of $\mathrm{ASH}^{10}{ }^{10}$ In the current patient, there was the presence of cross-fluctuation, but it was not transilluminating. This may be because of the muddy brownish fluid contents due to haemorrhage in the cyst. Other associated anomalies are contralateral hydrocele, hernia, ectopic testis, cryptorchidism and various urinary tract anomalies. In the current patient, there was no associated congenital anomaly.

Long-standing ASH may have complications such as testicular dysmorphism, hydronephrosis, lymphedema, pyocele, torsion, haematocele and rarely malignancy, which can be either testicular or paratesticular. $^{11-14}$

The presence of haematocele or bleeding inside the sac can occur and should raise the suspicion of the existence of malignancy. It can occur as an acute 
or chronic event and may be discovered accidentally. Haematocele can occur due to spontaneous rupture or trivial trauma or malignancy. ${ }^{13,14}$ The current patient had haematocele as a complication that probably developed over last two months as the patient presented with pain. In terms of diagnosis, ultrasound can provide information about the size and contents of the sac.

Computed tomography (CT) and magnetic resonance imaging (MRI) are additional investigations that can confirm the diagnosis and differentiate it from other diseases that mimic ASH. The presence of malignancy, associated congenital abnormality or presence of testicular dysmorphism, can be seen in $\mathrm{CT}$ and MRI. In the current case, there was a presence of testicular atrophy without any other congenital abnormality. The presence of a haematocele may not be diagnosed preoperatively, even with an imaging technique; often a haematocele will be diagnosed intraoperatively. In the current case, imaging was not suggestive of a haematocele and showed testicular atrophy.

ASH can be treated with a conservative or surgical approach. A conservative approach with the expectant spontaneous resolution has been seen in asymptomatic and uncomplicated paediatric ASH; still, such an approach is not useful in adults as spontaneous resolution does not occur in adults. Surgical procedures include excision of the sac, aspiration or incision and drainage. ${ }^{1}$ Excision of the sac is the ideal treatment option, which can be done laparoscopically or by open approach. A laparoscopic approach was first tried in 2004 and Boulhadiba et al. performed the first successful laparoscopic ASH excision in 2007. ${ }^{15}$ Excision of the abdominal sac along with partial excision of the scrotal sac with eversion is the most standard technique used. Sac can be excised en-bloc with cord and testis whenever there is suspicion of testicular atrophy or testicular malignancy. Partial excision of the scrotal sac with drainage of the abdominal sac can also be done. In the current patient, both sacs could be excised entirely with the removal of the left testis as there were testicular haematomas and atrophy.

Laparoscopy helps in confirming the diagnosis and is used in definitive management. The presence of large ASH and for a long period of time may make the laparoscopic treatment difficult due to adhesion or proximity to a vital structure, as was seen in the current case. Clinicians should be cautious with patients dealing with large ASH as open conversion may be required. This case is an example of complicated $\mathrm{ASH}$ where preoperative imaging studies did not show any evidence of complications within ASH. Even in complicated $\mathrm{ASH}$, the authors recommend laparoscopic treatment of ASH if the sacs can be excised safely. In addition, there may be a concomitant hernia in patients with ASH or it may develop in the future if the internal ring is dilated. Simultaneous repair of the dilated internal ring or hernia repair, if present, can prevent the future occurrence of a hernia.

\section{Conclusion}

$\mathrm{ASH}$ is a rare entity; although described mainly in the paediatric age group, it can also occur in adults as was seen in the current case. Clinical findings assisted by radiological imaging are the diagnostic modalities. ASH is usually asymptomatic and the development of symptoms is a sign of complicated ASH. Abdominoscrotal haematocele is a rare complication of ASH and to the best of the authors' knowledge, this is the first report to document such a case. Surgical excision is the definitive treatment in such cases and can be performed by an open or laparoscopic approach. The presence of a concomitant hernia or weak abdominal wall should be repaired at the same time. If there is a presence of only a dilated internal ring, it can be repaired at index surgery.

\section{AUTHORS' CONTRIBUTION}

OS and USK drafted the manuscript. SB collected the relevant data. USK conducted a critical revision of the manuscript. All authors approved the final version of the manuscript.

\section{References}

1. Gadelkareem RA. Abdominoscrotal hydrocele: A systematic review and proposed clinical grading. Afr J Urol 2018;24:83-92. https://doi.org/10.1016/j.afju.2018.01.006.

2. Ross JH, Kay R. Abdominoscrotal hydrocele in the infant. ClinPediatr (Phila) 1992;31:317-19. https://doi.org/10.1177/000992 289203100514

3. Mishra J, Behera TK, Rout B, Jena K, Ray Mohapatra CK. Hydrocele-en-bisac in a young adult: A rare encounter. ANZ J Surg 2013;83:581-2. https://doi.org/10.1111/ans.12220.

4. Dupuytren G. De l'hydrocèle et de sesprincipalesvariétés.In: Bailliêre G, Ed. Leçonsorales de cliniquechirurgicale. Paris, France. 1834. Pp 444-45.

5. Bickle LW. Abdominal or bilocular hydrocele. Br Med J 1919: $2 ; 13$.

6. Cuervo JL, Ibarra H, Molina M. Abdominoscrotal hydrocele: Its particular characteristics. J PediatrSurg 2009;44:1766-70. https://doi.org/10.1016/j.jpedsurg.2008.12.002

7. Singh D, Aga P, Goel A. Giant unilateral hydrocele "en-bisac" with right hydronephrosis in an adult: A rare entity. Indian J Urol2011;27:142-3. https://doi.org/10.4103/0970-1591.78413. 
8. Roller C. Abdominoscrotal hydrocele. JAMA 1934: 103;671-2. https://doi.org/10.1001/jama.1934.72750350001010

9. Celayir AC, Akyüz U, Ciftlik H, Gürbüz A, Danişmend N. A critical observation about the pathogenesis of abdominoscrotal hydroceles. J PediatrSurg 2001;36:1082-4. https://doi.org/10.10 53/jpsu.2001.24760.

10. Cozzi DA, Mele E, Ceccanti S, Pepino D, d'Ambrosio G, Cozzi F.Infantile abdominoscrotal hydrocele: A not so benign condition. J Urol2008;180:2611-15. https://doi.org/10.1016/j.ju ro.2008.08.054.

11. Avolio L, Chiari G, Caputo MA, Bragheri R. Abdominoscrotal hydrocele in childhood: Is it really a rare entity? Urology 2000;56:1047-9. https://doi.org/10.1016/s0090-4295(00)00801-3.
12. Belman AB.Abdominoscrotal hydrocele in infancy: A review and presentation of the scrotal approach for correction. J Urol 2001; 165:225-7. https://doi.org/10.1097/00005392-2001 01000-00065.

13. Estevão-CostaJ, Morgado $\mathrm{H}$, Soares-Oliveira $\mathrm{M}$, Campos $\mathrm{M}$, CarvalhoJL. Hemorrhagicabdominoscrotal hydrocele. A challenging entity. J PediatrSurg 2005;40:731-3. https://doi.org/10.1016/j. jpedsurg.2005.01.012.

14. Bisceglia M, Dor DB, Carosi I, Vairo M, Pasquinelli G. Paratesticular mesothelioma. Report of a case with comprehensive review of literature. AdvAnatPathol 2010;17:53-70. https://doi.org/10.109 7/PAP.0b013e3181c66fbc

15. Bouhadiba N, Godbole P, Marven S. Laparoscopic excision of abdominoscrotal hydrocele. J LaparoendoscAdvSurg Tech A 2007;17:701-3. https://doi.org/10.1089/lap.2006.0249. 